\title{
Waves and Turbulences in Solar and Stellar Atmospheres and Winds*)
}

\author{
Takeru K. SUZUKI and Takuma MATSUMOTO \\ Department of Physics, Nagoya University, Furo-cho, Chikusa, Nagoya 464-8602, Japan
}

(Received 6 December 2012 / Accepted 22 May 2013)

\begin{abstract}
Solar wind is the outflow of hot coronal plasma. Magnetohydrodynamical (MHD) wave and turbulence play an essential role in driving solar wind as well as stellar winds from solar-type stars. The sun, and in general, low-mass stars possess surface convective zones. Various modes of waves are excited from the stellar surfaces due to the convective motions, and these waves dissipate through various nonlinear processes such as turbulent cascade and the generation of compressive waves. As a result, the kinetic energy of the surface convection in the interior is transported to the upper atmosphere, which drives the wind. In this paper, we discuss these consecutive processes from both theoretical and observational viewpoints, and review our recent results of MHD simulations.
\end{abstract}

(C) 2013 The Japan Society of Plasma Science and Nuclear Fusion Research

Keywords: MHD, solar wind, turbulence, wave

DOI: $10.1585 /$ pfr.8.2401129

\section{Introduction}

The energy liberated via the $p+p$ chain nuclear fusion reaction in the central region of the sun is finally released by radiation from the photosphere. A tiny fraction, which is typically an order of $\sim 10^{-6}$, of the energy is transferred to the solar wind, the outflow emanating from the hot corona with temperature $\gtrsim 10^{6} \mathrm{~K}$, which is much larger than the temperature $(\approx 5780 \mathrm{~K})$ of the photosphere.

It is believed that the surface convective zone, in which the energy from the center is transferred outwardly by the motion of the gas itself, plays an essential role in driving the solar wind. Because of fluctuating motions involving the surface convection, various modes of waves are generated from the photosphere. The Alfvén wave, generated by the granulations or other surface activities, is a promising candidate operating in the heating and driving the solar wind. It can travel a long distance so that the dissipation plays a role in the heating of the solar wind plasma as well as the lower coronal plasma, in contrast to other processes, such as magnetic reconnection events and compressive waves [1], the heating of which probably concentrates at lower altitude.

Waves with the upgoing direction are supposed to be initially injected from the surface. Through the propagation, they suffer reflection, refraction, and various types of dissipation. As a result of these processes, the interplanetary space is mostly occupied by well-developed turbulence with both outgoing and incoming Alfvénic disturbances [2] (see Fig. 1). Since the Alfvén wave has energy flux, the dissipation of the outgoing component eventually leads to the heating and acceleration of the ambient

author'se-mail: stakeru@nagoya-u.jp

*) This article is based on the invited presentation at the 22nd International Toki Conference (ITC22).

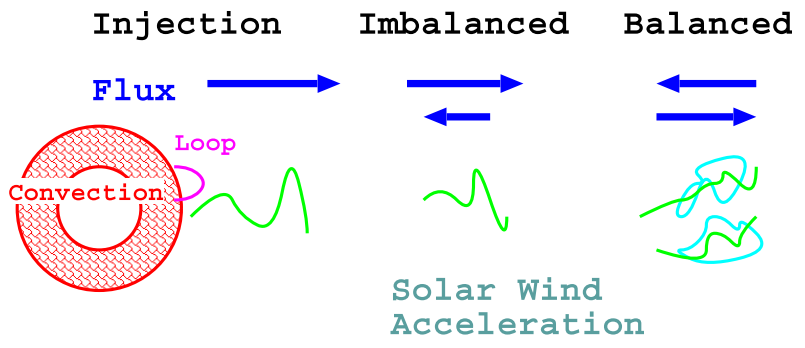

Fig. 1 A schematic picture for the energy transfer in the atmosphere of a solar-type star.

plasma. Various processes have been raised as candidates of the dissipation of the Alfvén waves, such as turbulent cascade [3-6], phase mixing [7], nonlinear mode conversion to compressive waves by Ponderomotive force [8-10] or by parametric decay [11-13], ioncyclotron resonance $[14,15]$, although there are still a lot of debates on the dominant process(es) which actually works in the sun.

The processes discussed so far is not unique in the sun. People believe that the stellar wind phenomena is universal in all the stars, and among them stars with a surface convection zone drive the stellar wind in a very similar manner to the sun. The main sequence stars with mass roughly below the solar mass, so-called solar-type stars, have a surface convection zone. Also, red giant stars generally posses a surface convection zone. In particular, the stellar wind from solar-type stars, which is important in terms of the formation and evolution of planets, is an active research field [16-18].

An important aspect of the plasma in the atmosphere of the sun and stars is that the density rapidly decreases with height because of the gravity by a star (Fig. 2). 


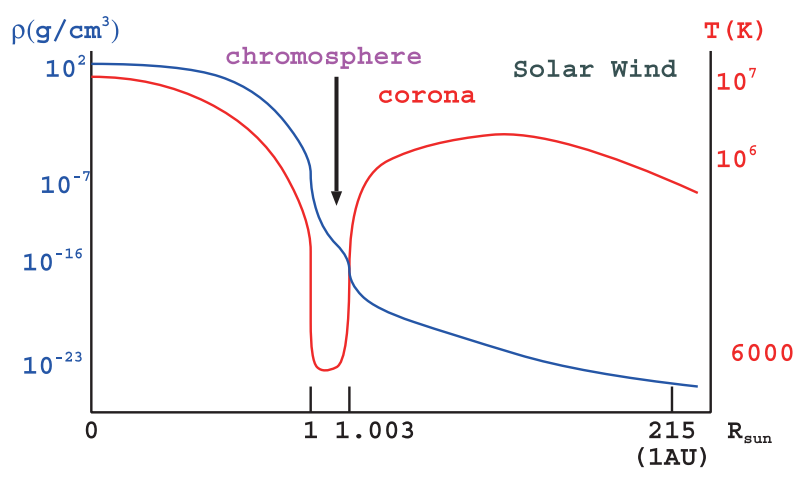

Fig. 2 Temperature (red; left axis) and density (blue; right axis) with astrocentric distance, $r$. The adopted values are typical for the sun. The vertical axis is normalized by the solar radius, $R_{\odot}=7 \times 10^{5} \mathrm{~km} ; r=1 R_{\odot}$ corresponds to the photosphere, and the corona is located typically above $r>1.003 R_{\odot}$, where the transition region is supposed to dynamically moves up and down. 1 astronomical unit (AU; Sun-Earth distance) equals to $215 R_{\odot}$.

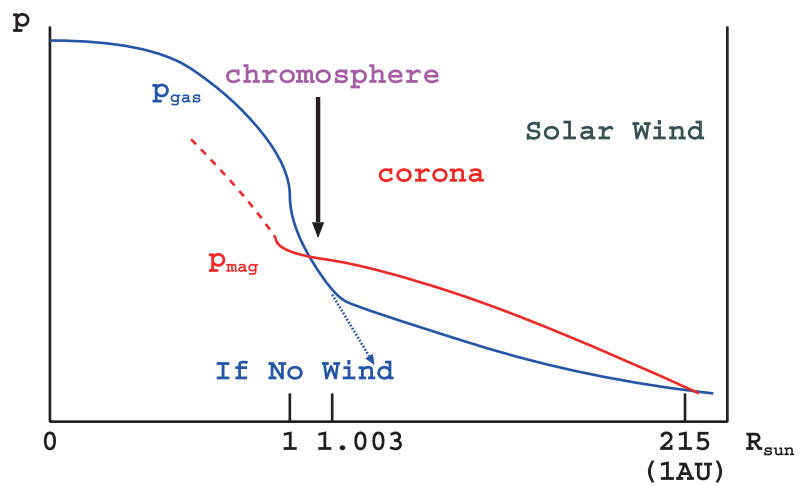

Fig. 3 Comparison of gas (blue) and magnetic (red) pressure in the atmosphere of a solar-type star. The scales are not precise. If the hydrostatic equilibrium (no solar wind) is satisfied, the gas pressure rapidly decreases in the atmosphere (blue dotted arrow), while in the realistic situation the change of the gas pressure is more gradual thanks to the redistribution of the density structure by the solar wind.

Assuming the hydrostatic equilibrium with the isothermal circumstances for simplicity, density, $\rho$ falls as $\rho \propto$ $\exp \left[-\frac{r-R_{\star}}{H}\right]$, where $r$ is astrocentric distance, $R_{\star}$ is a stellar radius and $H$ is a scale height. In general, $H$ is much smaller than $R_{\star}$, which leads to the rapid decrease of $\rho$. In the solar condition, the density in the low corona at $2000-10^{4} \mathrm{~km}$ ( $\ll$ the solar radius, $R_{\odot}=7 \times 10^{5} \mathrm{~km}$ ) is nearly 10 orders of magnitude lower than the density at the photosphere. The rapid change of the density gives the rapid change of the Alfvén speed, which triggers reflection as a consequence of the deformation of wave shape [19], which is discussed later based on our simulations.

The stratified atmosphere by the stellar gravity gives another important consequence, the dominance of the magnetic energy over other forms of energy, the kinetic ther-

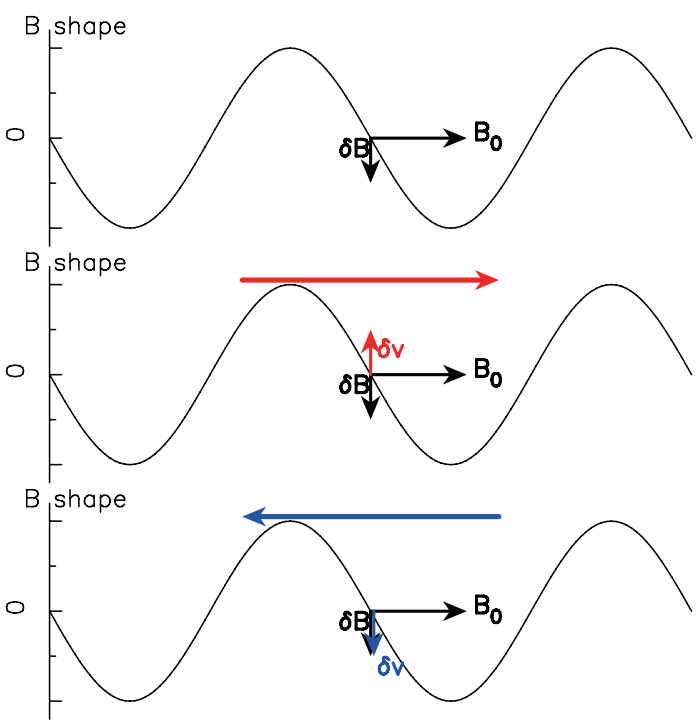

Fig. 4 Shape of a magnetic field line along which Alfvén wave is traveling. top: One cannot tell the propagation direction only from the shape. middle: If velocity perturbation, $\delta v$, and magnetic field perturbation, $\delta B$, are anti-correlated, the wave is traveling to the positive direction with background field, $B_{0}$. bottom: Similarly, if the $\delta v$ and $\delta B$ is correlated, the wave is propagating to the negative direction.

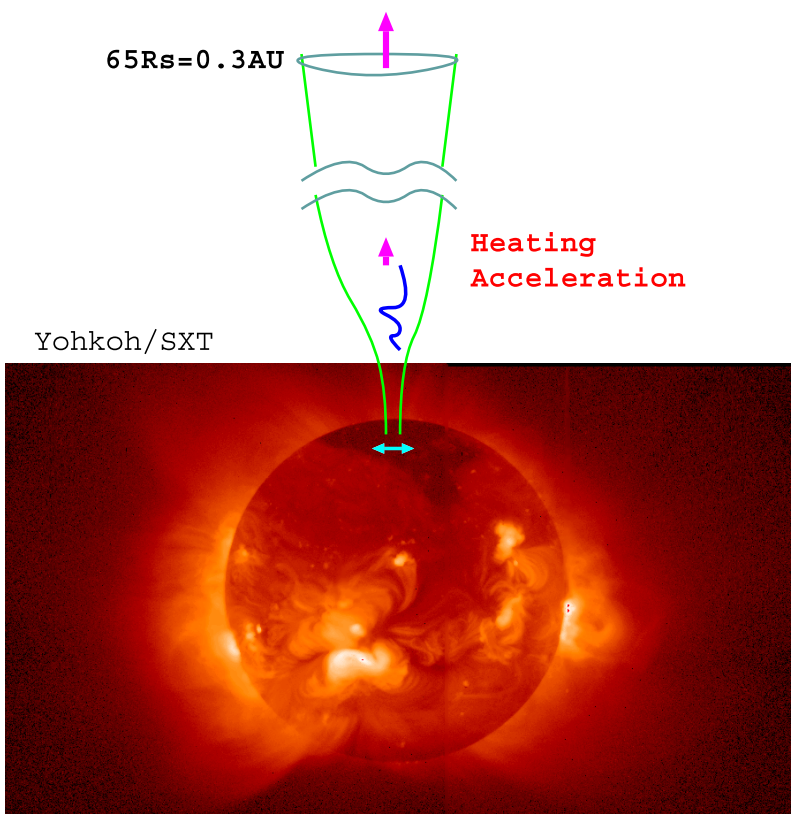

Fig. 5 An open flux tube from a coronal hole (the dark region in the polar region).

mal energy of the gas. In Fig. 3 we display schematic diagrams for the comparison of the magnetic and gas pressure as functions of $r$. The top panel is the condition where the background condition satisfies the hydrostatic equilibrium. In the solar condition, the average magnetic pressure is much smaller than the gas pressure at the photosphere, whereas magnetic pressure becomes comparable in local- 


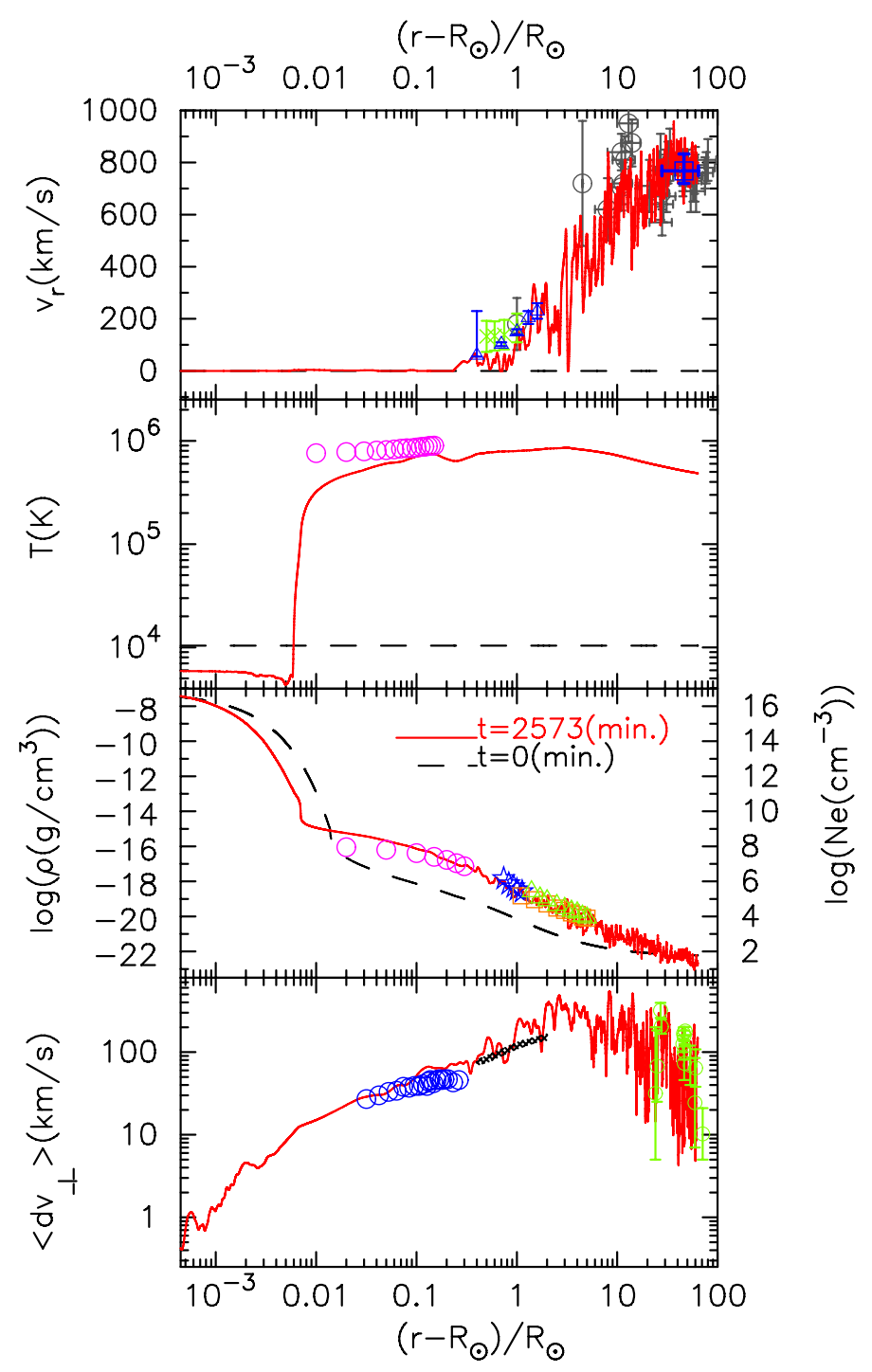

Fig. 6 Results of fast solar wind with observations in polar regions from our 1D simulation [9]. The figure is adopted from [9] with modification. From top to bottom, outflow speed, $v_{r}\left(\mathrm{~km} \mathrm{~s}^{-1}\right)$, temperature, $T(\mathrm{~K})$, density in logarithmic scale, $\log \left(\rho\left(\mathrm{g} \mathrm{cm}{ }^{-3}\right)\right)$, and rms transverse amplitude, $\left\langle d v_{\perp}\right\rangle\left(\mathrm{km} \mathrm{s}^{-1}\right)$ are plotted. Observational data in the third panel are electron density, $\log \left(N_{e}\left(\mathrm{~cm}^{-3}\right)\right)$ which is to be referred to the right axis. Dashed lines indicate the initial conditions and solid lines are the results at $t=2573$ minutes. In the bottom panel, the initial value $\left(\left\langle\delta v_{\perp}\right\rangle=0\right)$ does not appear. first: Green vertical error bars are proton outflow speeds in an interplume region by UVCS/SoHO [34]. Dark blue vertical error bars are proton outflow speeds by the Doppler dimming technique using UVCS/SoHO data [35]. A dark blue open square with errors is velocity by IPS measurements averaged in $0.13-0.3 \mathrm{AU}$ of high-latitude regions [36]. Light blue data are taken from [37]; crossed bars are IPS measurements by EISCAT, crossed bars with open circles are by VLBA measurements, and vertical error bars with open circles are data based on observation by SPARTAN 201-01 [38]. second: Pink circles are electron temperatures by CDS/SoHO [39]. third: Circles and stars are observations by SUMER/SoHO [40] and by CDS/SoHO [34], respectively. Triangles [34] and squares [41] are observations by LASCO/SoHO. fourth: Blue circles are non-thermal broadening inferred from SUMER/SoHO measurements [42]. Cross hatched region is an empirical constraint of non-thermal broadening based on UVCS/SoHO observation [43]. Green error bars are transverse velocity fluctuations derived from IPS measurements by EISCAT [44].

ized regions such as sun spots [20]. As moving upward, the gas pressure falls with decreasing density in an exponential manner. On the other hand, the decrease of the magnetic pressure is in a power-law manner; if a simple dipole geometry is assumed, $B \propto r^{-3}$, and accordingly the magnetic pressure falls with $r^{-6}$. Then, the decrease of the magnetic pressure is slower than the decrease of the gas pressure, and consequently the magnetic pressure dominates the gas pressure at a high altitude. Assuming the typical solar condition, the average magnetic pressure exceeds the gas pressure at $r \gtrsim 1000 \mathrm{~km}\left(\sim(1.001-1.002) R_{\odot}\right)$, corresponding to the chromospheric height. From the upper chromosphere to the coronal regions, the dynamics and energetics are basically controlled by the magnetic energy. This is a reason why the Alfvén wave plays a major role in driving the solar wind. 
When analyzing properties of Alfvén waves, Elsässer variables,

$$
z_{ \pm}=\delta v_{\perp} \mp \frac{\delta B_{\perp}}{\sqrt{4 \pi \rho}},
$$

is a useful index, where $\delta v_{\perp}$ and $\delta B_{\perp}$ are amplitudes of velocity and magnetic field associated with Alfvén wave, and $\perp$ indicates the perpendicular direction with respect to background field, $B_{0} . z_{+}\left(z_{-}\right)$denotes the amplitude of the Alfvén wave which travels to the (anti-)parallel direction with $B_{0}$ (Fig. 4). Elsässer variables are used to study the development of Alfvénic turbulence in solar wind plasma [21, 22]. Recently, Fujimura \& Tsuneta [23] observed the phase correlation between $\delta v_{\perp}$ and $\delta B_{\perp}$ at the photosphere, and found that reflected Alfvén waves from the upper region is so ubiquitous in the solar atmosphere, which we discuss from a theoretical point of view.

In this paper, we review our recent results of numerical simulations for the solar wind [9, 10, 24]. Although there are still uncertainties in the source region of the solar wind, a coronal hole, which are recognized as a dark area in a soft X-ray image as shown in Fig. 5 and roughly coincides with regions with magnetic field open to the interplanetary space [25], is believed to contribute to a sizable fraction of the total mass of the solar wind.

\section{Our Simulation}

\subsection{Setup}

We perform dynamical MHD simulations with radiative cooling and thermal conduction in a single flux tube in coronal holes, with focusing on the energy transfer. We started from one dimensional simulations $[9,10]$; we only consider $r$ dependence but treat 3 components of velocity and magnetic field. In two dimensional (2D) simulations [24], we take into account the dependences on one transverse component in addition to the $r$ dependence.

We adopt a super-radially open magnetic flux tube [26-28]. We initially set up a static and cool $\left(T=10^{4} \mathrm{~K}\right)$ atmosphere and start a simulation run by injecting velocity fluctuations from the photosphere with amplitude, $\delta v_{0} \sim 1 \mathrm{~km} \mathrm{~s}^{-1}$, comparable to the observed granule motions [29]. A great advantage of the code is that we can automatically determine mass loss rates as a dynamical output of the injected Poynting flux from the photosphere. For other details of the simulations, please consult $[9,10,24]$.

\subsection{Wind structure}

Figure 6 plots the initial condition (dashed lines) and the results after the quasi-steady state condition is achieved at $t=2573$ minutes (solid lines), compared with recent observations of fast solar winds. From top to bottom, $v_{r}\left(\mathrm{~km} \mathrm{~s}^{-1}\right), T(\mathrm{~K})$, mass density, $\rho\left(\mathrm{g} \mathrm{cm}^{-3}\right)$, and rms transverse amplitude, $\left\langle d v_{\perp}\right\rangle\left(\mathrm{km} \mathrm{s}^{-1}\right)$ are plotted. As for the density, we compare our result with observed electron density, $N_{e}$, in the corona. These variables are averaged for $3 \mathrm{~min}$ utes to incorporate observational exposure time. Figure 6

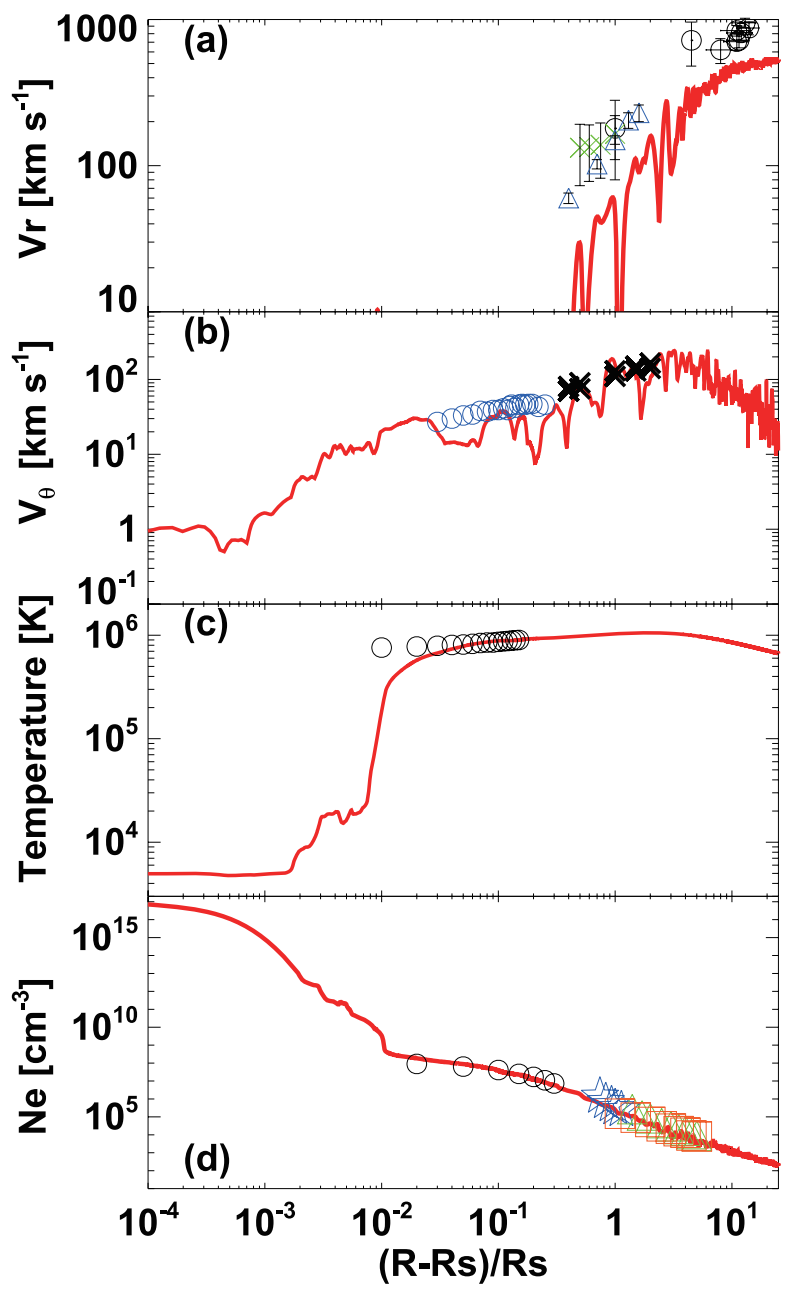

Fig. 7 The same as Fig. 6 but for our 2D simulation [24]. The figure is adopted from [24].

shows that the initially cool and static atmosphere is effectively heated and accelerated by the dissipation of the Alfvén waves. The sharp transition region which divides the cool chromosphere with $T \sim 10^{4} \mathrm{~K}$ and the hot corona with $T \sim 10^{6} \mathrm{~K}$ is formed owing to a thermally unstable region around $T \gtrsim 10^{5} \mathrm{~K}$ in the radiative cooling function [30,31]. The hot corona streams out as the transonic solar wind. The simulation naturally explains the observed trend quite well.

In Fig. 7, we show the result of the $2 \mathrm{D}$ simulation in comparison with observational data. The solid lines are the averaged over the transverse distance. The overall trend is quite similar to that of the 1D simulation, while the dissipation channels of the Alfvén waves is different as discussed later.

Figure 8 illustrates a snap-shot wind structure of the $2 \mathrm{D}$ simulation. The panels (a) and (b) show that density perturbations are stochastically excited depending on both radial and transverse directions. These density perturbations are mostly associated with compressive waves. The panels (c) and (d) indicates that the height of the transition region, which separates the cool chromosphere and the hot 


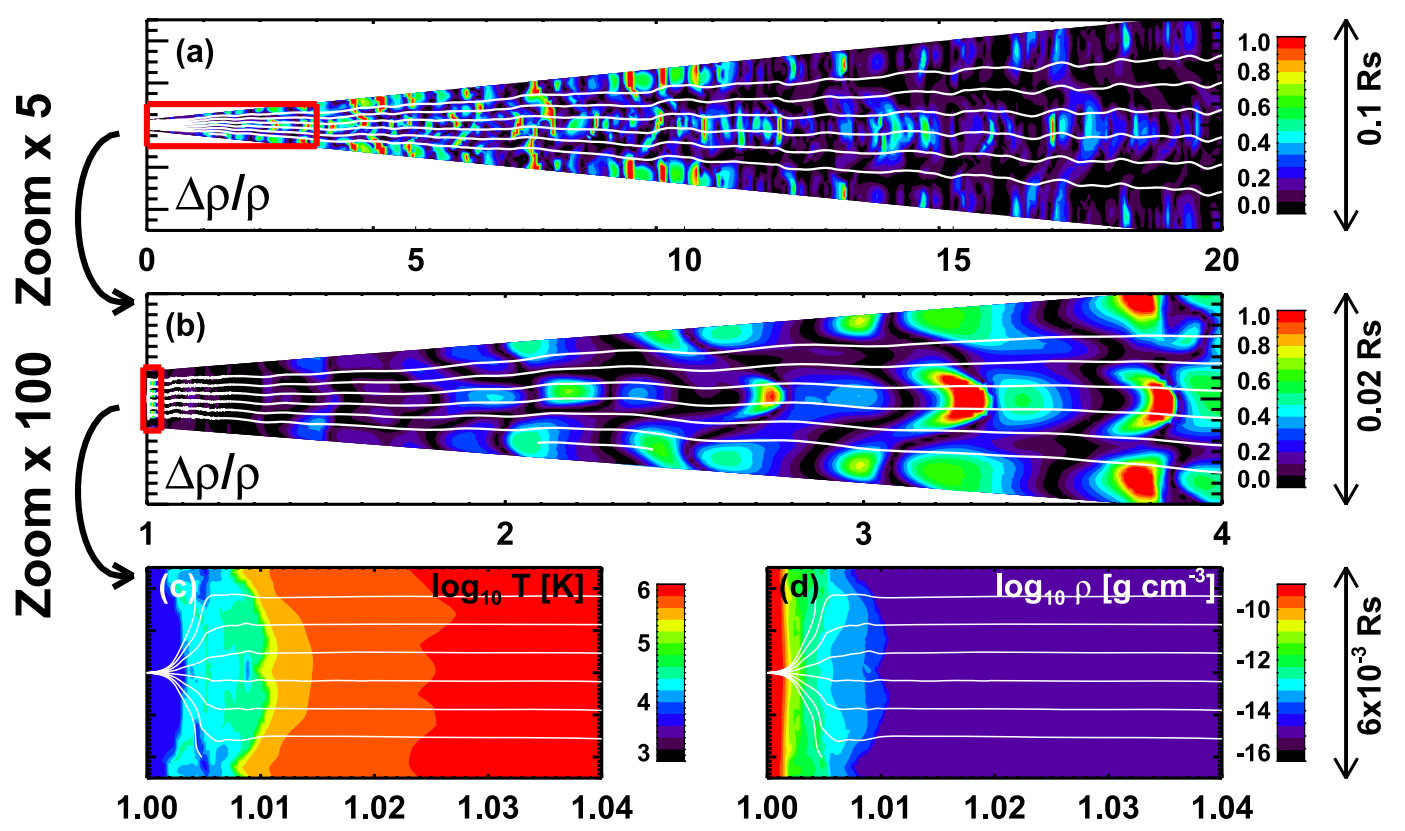

Fig. 8 A snapshot result of the 2D simulation from the photosphere to the interplanetary region. The figure is adopted from [24]. (a) Normalized density fluctuation, (b) region that is magnified 5 times. The red squared region in (a) is equivalent to (b). (c) Temperature distribution, (d) density distribution, the regions shown are magnified 100 times from (b). The red squared region in (b) is equivalent to (c) and (d). The white solid lines in each panel represent the magnetic field lines. Lengths are shown in units of the solar radius.

corona, varies with transverse distance. This clearly illustrates an importance of the 2D treatment, in contrast to the 1D treatment.

\subsection{Dissipation of Alfvén waves}

We investigate the dissipation of the Alfvén waves. The Alfvén waves which are generated from the photosphere suffer reflection before reaching the corona. In the chromosphere, which is located between the photosphere and the corona, the density decreases $\sim 8$ orders of magnitude, and as a result, the Alfvén speed largely increases. This triggers the deformation of the shape of propagating waves, and in the $1 \mathrm{D}$ and $2 \mathrm{D}$ simulations for the solar wind, $\sim 90 \%$ of the initial energy flux is reflected back downward $[9,10,24]$. This is consistent with the observation by [23], which reported that the amount of the downgoing Alfvénic disturbances is comparable to that of the outgoing component.

From now, we discuss properties of the Alfvén waves which transmit to the corona. We first start from discussions on the 1D simulation. Figure 9 presents radial distance - time diagrams of $v_{r}, \rho, v_{\perp}$, and $B_{\perp} / B_{r}$ in $R_{\odot} \leq r \leq$ $15 R_{\odot}$ from $t=2570 \mathrm{~min}$. to $2600 \mathrm{~min}$. of the $1 \mathrm{D}$ simulations. The fast MHD and Alfvén modes degenerate in our 1D treatment (wave vector and underlying magnetic field are in the same direction), so we simply call transverse waves Alfvén modes. In our simple 1D geometry, $v_{r}$ and $\rho$ trace the slow modes which have longitudinal wave components, while $v_{\perp}$ and $B_{\perp}$ trace the Alfvén modes which are transverse.

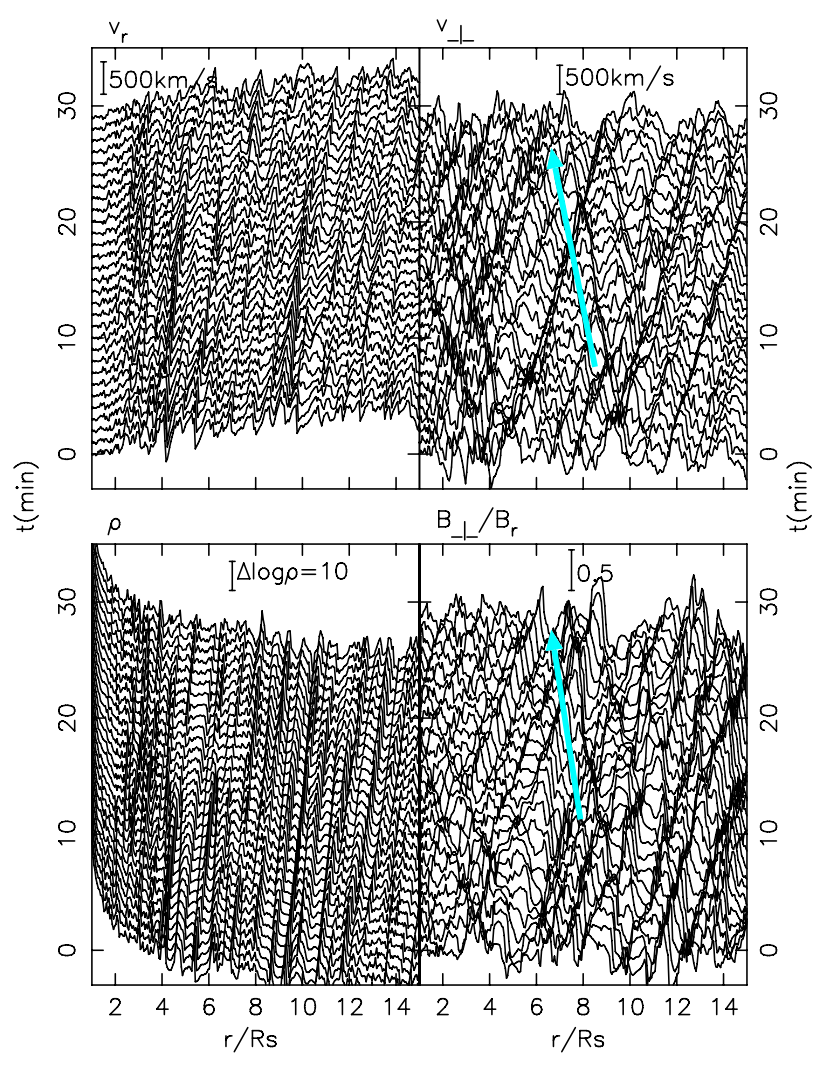

Fig. $9 \quad r-t$ diagrams for $v_{r}$ (upper-left), $\rho$ (lower-left), $v_{\perp}$ (upperright), and $B_{\perp} / B_{r}$ (lower-right.) The horizontal axises cover from $R_{\odot}$ to $15 R_{\odot}$, and the vertical axises cover from $t=2570$ minutes to 2600 minutes. In the upperright and lower-right panels, an example of an incoming Alfvén wave is shown by the arrows. 


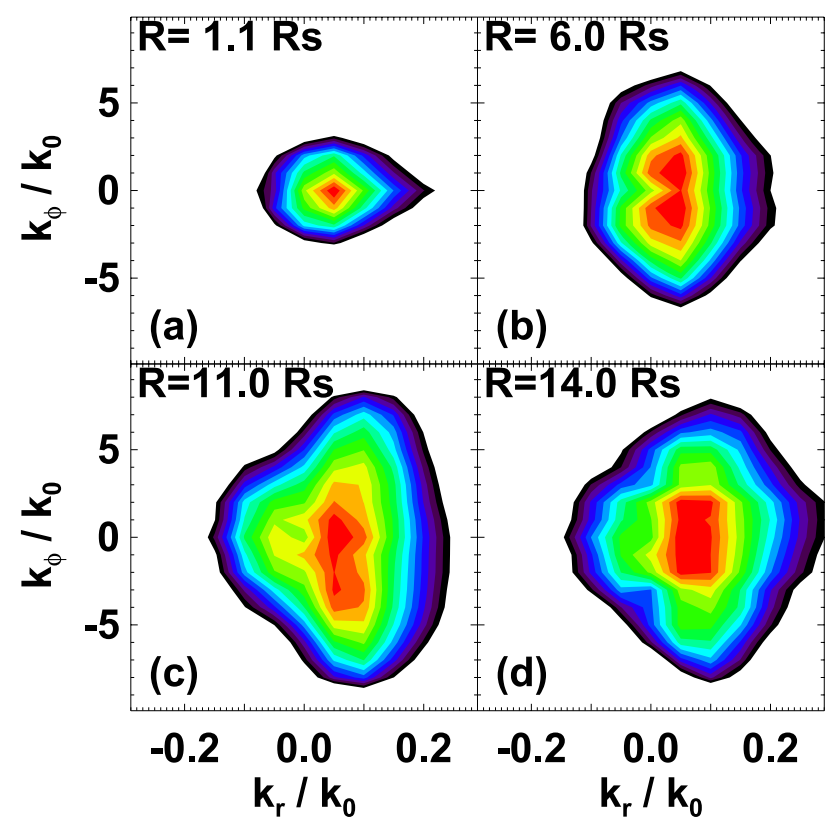

Fig. 10 Evolution of power spectrum of Alfvén wave as a function of radius. The figure is adopted from [24]. The four panels represent the power spectrum of Alfvén wave at (a) $R=1.1 R_{\odot}$, (b) $R=6.0 R_{\odot}$, (c) $R=11 R_{\odot}$, and (d) $R=14 R_{\odot}$. The vertical and the horizontal axis represents the wave number in $\phi$ direction, $k_{\phi}$, and in $\mathrm{r}$ direction, $k_{r}$, normalized by $k_{0}=2 \pi r \Delta \phi$, where $\Delta \phi$ is the angular system size in $\phi$ direction. The color in each panel shows the power spectral density in Fourier space normalized by the peak value.

One can clearly see the Alfvén waves in $v_{\perp}$ and $B_{\perp} / B_{r}$ diagrams, which have the same slopes with the Alfvén characteristics shown above. One can also find the incoming modes propagating from lower-right to upperleft as well as the outgoing modes generated from the surface. These incoming waves are generated by the reflection at the 'density mirrors' of the slow modes. At intersection points of the outgoing and incoming characteristics the non-linear wave-wave interactions take place, which play a role in the wave dissipation.

The slow modes are seen in $v_{r}$ and $\rho$ diagrams. Although it might be difficult to distinguish, most of the patterns are due to the outgoing slow modes ${ }^{1}$ which are generated from the perturbations of the Alfvén wave pressure, $B_{\perp}^{2} / 8 \pi[8]$. These slow waves steepen eventually and lead to the shock dissipation. The main channel of the dissipation of the outgoing Alfvén waves is the nonlinear generation of compressive waves in our 1D treatment.

In the 2D simulation, we can treat turbulence phenomena in addition to compressive waves, whereas the turbulence in 2D geometry is still different from the turbulence in realistic 3D geometry. Figure 10 exhibits the evolu-

\footnotetext{
${ }^{1}$ The phase correlation of the longitudinal slow waves is opposite to that of the transverse Alfvén waves. The outgoing slow modes have the positive correlation between amplitudes of $v_{r}$ and $\rho,\left(\delta v_{r} \delta \rho>0\right)$, while the incoming modes have the negative correlation $\left(\delta v_{r} \delta \rho<0\right)$.
}

tion of power spectrum of the Alfvén waves. The 4 panels clearly show that the Alfvénic perturbations preferentially evolve to the perpendicular direction with respect to the background radial magnetic field from the surface to the upper locations [32].

We estimate the heating as a result of electric currents, which contains the turbulent cascade, in comparison with the heating carried by the dissipation of the nonlinearly generated compressive waves [24,33]. We found that the heating by the currents partly dominate the heating by the compressive waves. Realistic 3D treatment of the solar wind is required to pin down the actual dissipation mechanism of the Alfvén waves.

Numerical computations were carried out on Cray XT4 at Center for Computational Astrophysics, CfCA, of National Astronomical Observatory of Japan. Takeru K. Suzuki is supported by Grants-in-Aid for Scientific Research from the MEXT of Japan, 22864006. Takuma Matsumoto gratefully acknowledges the research support in the form of fellowship from the Japan Society for the Promotion of Science for Young Scientists.

[1] T.K. Suzuki, Astrophys. J. 578, 598 (2002).

[2] R. Bruno and V. Carbone, Living Review of Solar Physics 2, 4 (2005).

[3] W.H. Matthaeus, G.P. Zank, S. Oughton, D.J. Mullan and P. Dmitruk, Astrophys. J. Lett. 523, L93 (1999).

[4] B.D.G. Chandran, Phys. Rev. Lett. 95, 5004 (2005).

[5] S.R. Cranmer, A.A. van Ballegooijen and R.J. Edgar, Astrophys. J. Suppl. 171, 520 (2007).

[6] A. Verdini and M. Velli, Astrophys. J. 662, 669 (2007).

[7] J. Heyvaerts and E.R. Priest, Astron. Astronomy 117, 220 (1983).

[8] T. Kudoh and K. Shibata, Astrophys. J. 514, 493 (1999).

[9] T.K. Suzuki and S. Inutsuka, Astrophys. J. Lett. 632, L49 (2005).

[10] T.K. Suzuki and S. Inutsuka, J. Geophys. Res. 111, A06101 (2006).

[11] M.L. Goldstein, Astrophys. J. 219, 700 (1978).

[12] T. Terasawa, M. Hoshino, J.I. Sakai and T. Hada, J. Geophys. Res. 91, 4171 (1986).

[13] Y. Nariyuki, T. Hada and K. Tsubouchi, J. Geophys. Res. 114, A07102 (2009).

[14] W.I. Axford and J.F. McKenzie, The Solar Wind in "Cosmic Winds and the Heliosphere", Eds. J.R. Jokipii, C.P. Sonnet and M.S. Giampapa (University of Arizona Press, 1997).

[15] C.Y. Tu and E. Marsch, Astron. Astrophys. 368, 1071 (2001).

[16] B.E. Wood, H.-R. Müller, G.P. Zank and J.L. Linsky, Astrophys. J. 574, 412 (2002).

[17] B.E. Wood, H.-R. Müller, G.P. Zank, J.K. Linsky, S. Redfield, Astrophys. J. Lett. 628, L143 (2005).

[18] T.K. Suzuki et al., PASJ 65, in press (2013).

[19] R.L. Moore, S.T. Suess, Z.E. Musielak and A.-H. An, Astrophys. J. 378, 347 (1991).

[20] Y. Katsukawa and S. Tsuneta, Astrophys. J. 621, 498 (2005).

[21] B. Bavassano, E. Pietropaolo and R. Bruno, J. Geophys. Res. 106, 10659 (2001).

[22] N. Yokoi and F. Hamba, Phys. Plasmas 14, 112904 (2007).

[23] D. Fujimura and S. Tsuneta, Astrophys. J. 702, 1443 
(2009).

[24] T. Mastumoto and T.K. Suzuki, Astrophys. J. 749, 8 (2012).

[25] K. Hakamada, M. Kojima, T. Ohmi, M. Tokumaru and K. Fujiki, Sol. Phys. 227, 387 (2005)

[26] S. Tsuneta et al., Astrophys. J. 688, 1374 (2008).

[27] H. Ito, S. Tsuneta, D. Shiota, M. Tokumaru and K. Fujiki, Astrophys. J. 719, 131 (2010).

[28] D. Shiota, S. Tsuneta, M. Shimojo, N. Sako, D. Orozco Suórez and R. Ishikawa, Astrophys. J. 753, 157 (2012).

[29] T. Matsumoto and R. Kitai, Astrophys. J. Lett. 716, L19 (2010).

[30] M. Landini and B.C. Monsignori-Fossi, Astron. Astrophys. Supp. 82, 229 (1990).

[31] R.S. Sutherland and M.A. Dopita, Astrophys. J. Suppl. 88, 253 (1993).

[32] P. Goldreich and S. Sridhar, Astrophys. J. 438, 763 (1995).

[33] T. Matsumoto and T.K. Suzuki, submitted to MNRAS (2013).

[34] L. Teriaca, G. Poletto, M. Romoli and D.A. Biesecker, Astrophys. J. 588, 566 (2003).

[35] L. Zangrilli, G. Poletto, P. Nicolosi, G. Noci and M. Romoli, Astrophys. J. 574, 477 (2002).

[36] M. Kojima, A.R. Breen, K. Fujiki, K. Hayashi, T. Ohmi and M. Tokumaru, J. Geophys. Res. 109, A04103 (2004).

[37] R.R. Grall, W.A. Coles, M.T. Klinglesmith, A.R. Breen, P.J.S. Williams, J. Markkanen and R. Esser, Nature 379, 429 (1996).

[38] S.R. Habbal, R. Esser, M. Guhathakura and R.R. Fisher, Gephys. Res. Lett. 22, 1465 (1994).

[39] A. Fludra, G. Del Zanna and B.J.I. Bromage, Spa. Sci. Rev. 87, 185 (1999).

[40] K. Wilhelm, E. Marsch, B.N. Dwivedi, D.M. Hassler, P. Lemaire, A.H. Gabriel and M.C.E. Huber, Astrophys. J. 500, 1023 (1998).

[41] P. Lamy, E. Quemerais, A. Liebaria, M. Bout, R. Howard, R. Schwenn and G. Simnett, in Fifth SOHO Worshop, The Corona and Solar Wind near Minimum Activity, ed A. Wilson (ESA-SP 404; Noordwijk:ESA), 491 (1997).

[42] D. Banerjee, L. Teriaca, J.G. Doyle and K. Wilhelm, Astron. Astrophys. 339, 208 (1998).

[43] R. Esser, S. Fineschi, D. Dobrzycka, S.R. Habbal, R.J. Edgar, J.C. Raymond and J.L. Kohl, Astrophys, J. Lett. 510, L63 (1999).

[44] A. Canals, A.R. Breen, L. Ofman, P.J. Moran and R.A. Fallows, Ann. Geophys. 20, 1265 (2002). 